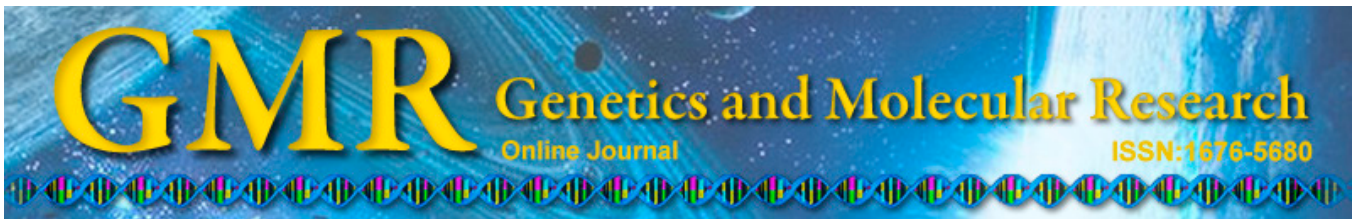

\title{
Involvement of AP-1 in p38MAPK signaling pathway in osteoblast apoptosis induced by high glucose
}

\author{
Z.P. Feng, H.C. Deng, R. Jiang, J. Du and D.Y. Cheng \\ Department of Endocrinology, The First Affiliated Hospital, \\ Chongqing Medical University, Chongqing, China \\ Corresponding author: Z.P. Feng \\ E-mail: fengzhengping_f@yeah.net
}

Genet. Mol. Res. 14 (2): 3149-3159 (2015)

Received August 12, 2014

Accepted November 17, 2014

Published April 10, 2015

DOI http://dx.doi.org/10.4238/2015.April.10.26

\begin{abstract}
We investigated the effect of p38MAPK/AP-1 (activator protein-1) signaling on the apoptosis of osteoblasts induced by high glucose. A lentivirus vector of small hairpin RNA (shRNA) targeting $\mathrm{p} 38 \mathrm{MAPK}$ was constructed in vitro. Osteoblasts MC3T3-E1 cultured in vitro were treated with vehicle, high glucose, p38MAPKshRNA transfection, p38MAPK inhibitor, and unrelated shRNA transfection. Apoptosis, protein levels of p38MAPK, and activities of AP-1 in MC3T3-E1 osteoblasts were measured using TUNEL and flow cytometry, Western blot analysis, and an electrophoretic mobility shift assay. Compared with the vehicle group, high glucose induced apoptosis of MC3T3-E1 osteoblasts and activated p38MAPK and AP1. p38MAPK-shRNA transfection blocked the effect of high glucose stimulation, and the p38MAPK inhibitor showed similar effects as those observed in p38MAPK transfection. Unrelated shRNA had no effect on these changes in MC3T3-E1 osteoblasts induced by high glucose. Therefore, our results suggest that p38MAPK-shRNA can
\end{abstract}


reduce apoptosis of MC3T3-E1 osteoblasts induced by high glucose by inhibiting the p38MAPK-AP-1 signaling pathway.

Key words: p38MAPK; AP-1; MC3T3-E1 osteoblast; RNA interference; Apoptosis

\section{INTRODUCTION}

Osteoporosis and diabetes mellitus (DM) are chronic metabolic diseases that threaten human health (Blakytny et al., 2011). Since Albright first proposed in 1948 that DM could result in osteal changes, additional studies were conducted to examine the mechanism of osteal changes induced by DM to prevent diabetic osteoporosis and fracture. DM induces bone formation defects, demonstrated by reduced osteoblast numbers, deficient osteoid formation, and slowed mineralization of bone (Inzerillo and Epstein, 2004). Previous studies (Shanmugam et al., 2003; Alikhani et al., 2007; Qi et al., 2008) have indicated that the occurrence of diabetic osteopathia is closely correlated with apoptosis of skeletal system cells and that long-term hyperglycemia in diabetic patients increases the production of advanced glycation end-products (AGEs). These AGEs bind to the AGE receptor on the surface of cells to stimulate secretion of cytokines, including tumor necrosis factor, interleukin-6, and interleukin-1 to promote the apoptosis of osteoblasts and finally reduce the number of osteoblasts and volume of bone formation (Shanmugam et al., 2003; Alikhani et al., 2007; Qi et al., 2008). Although osteoblast apoptosis is thought to play an important role in diabetic osteoporosis as a key link, the molecular mechanism of this process is not clear.

As a common pathway of intracellular signaling transduction after extracellular stimulation, signal transduction of p38 mitogen-activated protein kinase (p38MAPK) is closely correlated with cellular growth, development, proliferation, and apoptosis (Tsuchiya et al., 2008). As a downstream transcription factor of the MAPK signaling pathway, activator protein-1 (AP-1) has received increasing attention. AP-1 is a bZIP-type DNA binding protein and is composed of Jun and Fos family members; Jun and Fos can form homogeneous or heterogeneous dimmers or bind to other transcriptional factors and pro-oncogenes such as nuclear factor-kB, bcl-2, and nuclear hormone receptors to modulate the transcription of several cytokines (Smeyne et al., 1993; Liu et al., 1998; Nicodemo et al., 1998). In addition, c-fos and c-jun are thought to be involved in chondrocyte proliferation, gene expression of bone matrix protein, and osteoblast activation (Oyama et al., 1998). In primary osteoporosis, expression of c-fos and c-jun is increased and is related to apoptosis of osteoblasts, suggesting the involvement of AP-1 (Lin et al., 2003). Our previous study indicated that high glucose can activate the p38MAPK signaling pathway to induce osteoblast apoptosis by modulating caspase- 1 and bcl-2 (Feng et al., 2011), suggesting that the p38MAPK is an initiating factor for diabetic osteopathia and may modulate diabetic osteoporosis through AP-1. However, whether AP-1 is involved in high glucose induced osteoblast apoptosis is unclear. In the present study, we investigated whether activation of p38MAPK under highglucose conditions increases AP-1 activity and induces osteoblast apoptosis, to provide a theoretical basis for the treatment of diabetic osteopathia. 


\section{MATERIAL AND METHODS}

\section{Preparation of p38MAPK-shRNA (small hairpin RNA) lentivirus vector}

Based on the gene sequence of p38MAPK in GenBank and the design principle of Reynolds, online software was used to screen 3 interference target sequences: siRNA1, 5'-CTCAGAGTCTGCAAGAAACTA-3'; siRNA2, 5'-CCAACAATTCTGCTCTGGTTA-3'; siRNA3, 5'-AGAGTCTGCAAGAAACTACAT-3'; and nonspecific sequence, 5'-GACTTCATA AGGCGCATGC-3'. Synthetic sense and anti-sense strands were annealed to form the shRNA template and ligated into the P113.7 vector, which had been subjected to double-digestion with $\mathrm{HpaI} / \mathrm{XhoI}$ and linearized. Harvested plasmids were referred to as p38MAPK-shRNA1, p38MAPK-shRNA2, and p38MAPK-shRNA3, respectively. The lentivirus shuttle plasmid and auxiliary packaging vector plasmid were prepared for co-transfection of 293T cells (SIBS; Shanghai, China). The supernatant containing enriched lentivirus particles was collected for transfection of HeLa cells; virus titer was measured using flow cytometry.

\section{MC3T3-E1 osteoblast culture and lentivirus transfection}

MC3T3-E1 osteoblasts (American Type Culture Collection, Manassas, VA, USA) were cultured in $\alpha$-MEM supplemented with $10 \%$ fetal bovine serum, $100 \mathrm{U} / \mathrm{mL}$ penicillin, and $100 \mathrm{U} / \mathrm{mL}$ streptomycin (Gibco, Grand Island, NY, USA), and propagated for passages with regular medium changes. When confluence reached 70-90\%, MC3T3-E1 cells were transfected with the lentivirus solution. MC3T3-E1 osteoblasts were grouped as a normal control group (cultured with $5.5 \mathrm{mM}$ D-glucose for 7 days), high-glucose group (cultured with $22.2 \mathrm{mM}$ D-glucose for 7 days), p38MAPK-shRNA transfection group (transfected with p38MAPK-shRNA1-3 for $24 \mathrm{~h}$ and cultured with $22.2 \mathrm{mM}$ D-glucose for 7 days), p38MAPK inhibitor group (pre-cultured with $20.0 \mu \mathrm{M}$ p38MAPK inhibitor (SB203580) for $2 \mathrm{~h}$ and followed by culture with $22.2 \mathrm{mM}$ D-glucose for 7 days), and unrelated shRNA transfection group (cultured with blank vector for $24 \mathrm{~h}$ followed by culture with $22.2 \mathrm{mM} \mathrm{D}$-glucose for 7 days). Each group consisted of 3 wells.

\section{Measurement of p38MAPK mRNA expression in MC3T3-E1 cells with fluorescence real-time polymerase chain reaction (PCR)}

Total RNAs were extracted according to the Trizol manual (Invitrogen, Carlsbad, CA, USA), and was used for reverse transcription as a template. p38MAPK primers were designed according to the mouse p38MAPK gene sequence using the Clone manager software; primers were synthesized by Invitrogen (Table 1). The $\beta$-actin gene was used as an internal control.

After amplification with HS Taq polymerase (Invitrogen), PCR products were analyzed using software to measure the fluorescence threshold cycle $(\mathrm{Ct})$, which was compared with the standard curve to calculate the p38MAPK copy number and expression levels. The formula for inhibition rate was as follows: inhibition rate $=$ (control group - interference group) / control group x 100\%. 
Table 1. PCR primers of the $\mathrm{p} 38 \mathrm{MAPK}$ gene.

\begin{tabular}{llc}
\hline Gene & Primer sequence & Production segment (bp) \\
\hline$\beta$-actin & Primer 1: 5'-AGGTGACAGCATTGCTTCTG-3' & 188 \\
p38MAPK & Primer 2: 5'-GCTGCCTCAACACCTCAAC-3' & 398 \\
& Primer 1: 5'-GCTGAACAAAGGGAGAGACG-3' & 398 \\
\hline
\end{tabular}

\section{Measurement of MC3T3-E1 apoptosis using TUNEL and flow cytometry}

Regular cell smears were fixed with 4\% paraformaldehyde, digested with protein kinase $\mathrm{K}$, mixed with TUNEL solution, and incubated in humidified atmosphere in the dark at $37^{\circ} \mathrm{C}$ for $1 \mathrm{~h}$. Finally, slides were subjected to color development with $40 \mu \mathrm{L}$ streptavidin-conjugated horseradish peroxidase (HRP), $0.04 \% 3$,3'-diaminobenzidine, and $0.03 \% \mathrm{H}_{2} \mathrm{O}_{2}$ for 10 min, and mounted with regular resin. The nuclei of apoptotic MC3T3-E1 were stained tan or brown. Ten fields under high magnification were randomly selected and 100 cells in each field were counted to determine the apoptotic index using the following formula: TUNEL positive cell number / total cell number x $100 \%$.

\section{Measurement of p38MAPK proteins in MC3T3-E1 osteoblasts by Western blot}

MC3T3-E1 cells were collected to extract proteins and measure protein concentration using a Coomassie Blue staining kit (Invitrogen). A total of $50 \mu \mathrm{g}$ protein was subjected to sodium dodecyl sulfate-polyacrylamide gel electrophoresis (SDS-PAGE) and transferred to a polyvinylidene fluoride membrane, which was blocked with phosphate-buffered saline (PBS) containing 5\% skim milk for $6 \mathrm{~h}$. Rabbit anti-mouse p38MAPK antibody (1:300; Santa Cruz Biotechnology, Santa Cruz, CA, USA) and monoclonal GAPDH antibody (1:300; Santa Cruz) were added, and incubated for $12 \mathrm{~h}$ at $4^{\circ} \mathrm{C}$. HRP-conjugated sheep anti-rabbit Ig $(1: 10,000$; Santa Cruz Biotechnology) was added and the membrane was incubated for $2 \mathrm{~h}$. Enhanced chemiluminescence solution was added for development. A gel imaging system was used to analyze the gray value.

\section{Measurement of AP-1 activities in MC3T3-1 osteoblasts with electrophoretic mobility shift assay (EMSA)}

Collected MC3T3-1 osteoblasts were placed in $500 \mu \mathrm{L}$ pre-cooled nucleoprotein extracting solution A to extract nucleoprotein and measure protein content. After complete mixing for $10 \mathrm{~min}$ at $37^{\circ} \mathrm{C}$, each nucleoprotein sample was mixed with $1 \mu \mathrm{L}$ biotin-labeled AP-1 probe that had a sequence of 5'-CGCTTGATGAGTCAGCCGGAA-3' and was subjected to PAGE. Next, the nucleoprotein was transferred from the gel to a membrane, which was ultraviolet cross-linked for 5 min and blocked with 5\% bovine serum albumin for $1 \mathrm{~h}$. Subsequently, the membrane was incubated with streptavidin-HRP (1:1000; Invitrogen) on a shaking bed at $37^{\circ} \mathrm{C}$ for $1 \mathrm{~h}$ and rinsed with PBS 4 times for 5 min each time. Finally, chemiluminescence substrate was added to the membrane in a dark room for $5 \mathrm{~min}$ and developed for analysis. The experiment was repeated 3 times. 


\section{Statistical analysis}

All data are reported as means \pm standard deviation (SD), and the difference between groups was analyzed using one-way analysis of variance using the SPSS 11.0 software (SPSS, Inc., Chicago, IL, USA). P $<0.05$ was considered to be significant.

\section{RESULTS}

\section{Inhibition of p38MAPK mRNA in MC3T3-E1 cells by transfection of p38MAPK-shRNA}

The results indicated that there was no significant difference between the normal control group and unrelated shRNA transfection group regarding the expression of p38MAPK in MC3T3-E1 cells $(P>0.05)$. Expression of p38MAPK in the p38MAPK-shRNA1, p38MAPKshRNA2, and p38MAPK-shRNA3 groups was decreased by $78.8,84.3$, and $60.2 \%$, respectively. These differences were significant when compared with the normal control group $(\mathrm{P}<0.01)$, with a maximum inhibitory effect observed in the p38MAPK-shRNA2 group, as shown in Figure 1A-C.

A

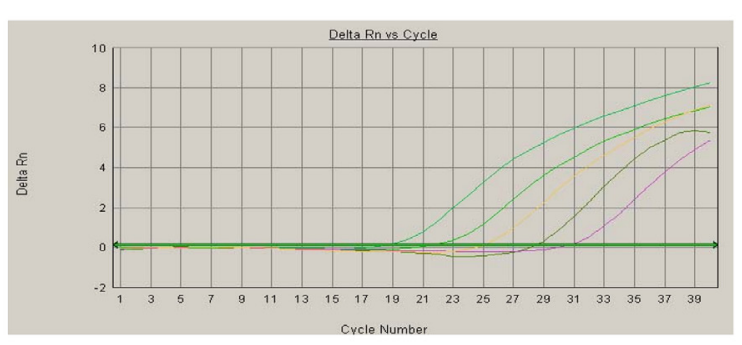

B

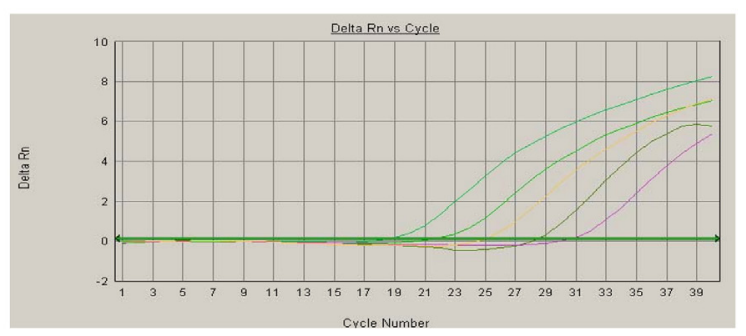

C

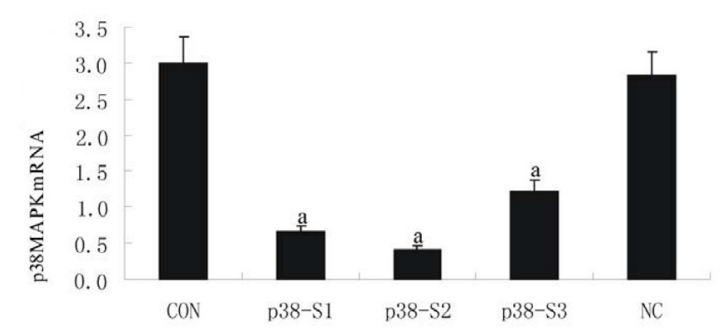

Figure 1. Inhibitory effect of p38MAPK-shRNA on the expression of p38MAPK mRNA. A. Standard curve; B. amplification curve of p38MAPK in different groups; C. relative expression of p38MAPK in different groups. Con $=$ normal control; p38-S1 = p38MAPK-shRNA1; p38-S2 = p38MAPK-shRNA2; p38-S3 = p38MAPK-shRNA; NC = unrelated p38MAPK-shRNA transfection. ${ }^{a} \mathrm{P}<0.01$ vs Con. 


\section{Effect of p38MAPK inhibition on apoptosis of MC3T3-E1 osteoblasts}

As shown in Figure 2, apoptotic MC3T3-E1 cells induced by high glucose appeared as tan or brown in color following TUNEL staining; they also showed a reduced microvilli, incomplete cellular membrane, swollen mitochondria, expanded endoplasmic reticulum, and marginalized chromosome. When compared with the normal control group (Figure 2A), apoptosis of MC3T3-E1 osteoblasts in the high-glucose group (Figure 2B) and unrelated shRNA transfection group (Figure 2C) was significantly increased $(\mathrm{P}<0.01$; Figure $2 \mathrm{D}$ and Table 2), whereas apoptosis of MC3T3-E1 osteoblasts in the p38MAPK-shRNA transfection group was significantly decreased $(\mathrm{P}<0.05$; Figure $2 \mathrm{E}, \mathrm{F}$ and Table 2$)$. Similarly, the p38MAPK inhibitor SB203580 significantly decreased the apoptosis of MC3T3-E1 osteoblasts $(\mathrm{P}<0.01$; Figure 2F and Table 2).

A

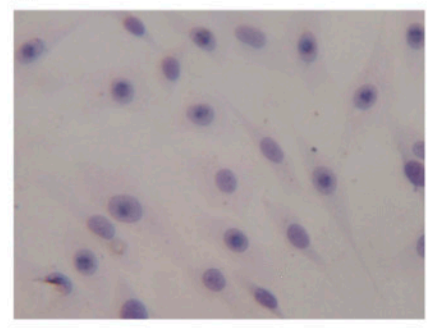

D

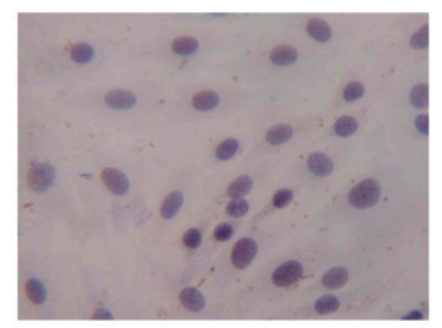

B

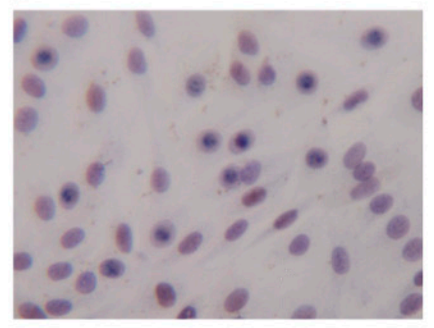

$E$

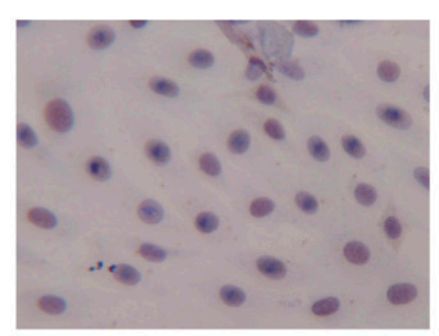

C
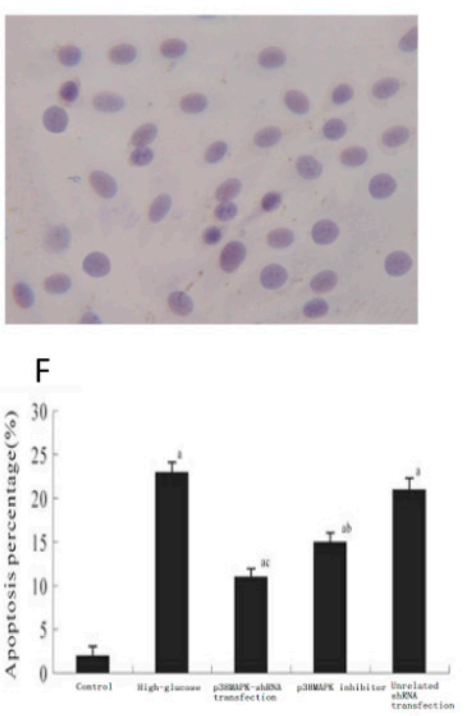

Figure 2. Effect of p38MAPK-shRNA transfection inhibitor on apoptosis of MC3T3-E1 osteoblasts. A. Control; B. high-glucose; C. p38MAPK-shRNA transfection; D. p38MAPK inhibitor; E. unrelated p38MAPK-shRNA transfection; F. apoptosis percentage in different groups. ${ }^{a} \mathrm{P}<0.01 v s$ control; ${ }^{\text {b }} \mathrm{P}<0.05 v s$ high-glucose group; ${ }^{\mathrm{c}} \mathrm{P}<0.01$ vs high-glucose group.

Table 2. Positive expression of TUNEL in MC3T3-E1 cells.

\begin{tabular}{lr}
\hline Group & TUNEL positive rate (\%) \\
\hline Normal control & $3.15 \pm 0.68$ \\
High-glucose & $16.36 \pm 3.24^{\Delta}$ \\
p38MAPK-shRNA transfection & $8.98 \pm 1.26^{\Delta * *}$ \\
p38MAPK inhibitor & $11.42 \pm 2.75^{\Delta *}$ \\
Negative shRNA transfection & $17.24 \pm 2.90^{\Delta}$ \\
\hline
\end{tabular}

Data are reported as means $\pm \mathrm{SD}$ for $\mathrm{N}=3 .{ }^{\Delta} \mathrm{P}<0.01$ vs normal control, $* \mathrm{P}<0.05$ vs high-glucose; $* * \mathrm{P}<0.01$ vs high-glucose. 
Flow cytometry showed similar results (Table 3 ). When compared with the normal control group, apoptosis of MC3T3-E1 osteoblasts in the high-glucose group and unrelated p38MAPKshRNA transfection group significantly increased $(\mathrm{P}<0.01)$. By contrast, apoptosis of MC3T3-E1 cells in the p38MAPK-shRNA transfection group and p38MAPK inhibitor group was significantly reduced by $50.8 \%(\mathrm{P}<0.01)$ and $37.5 \%(\mathrm{P}<0.05)$, respectively, when compared with levels in the high-glucose group. Furthermore, a reduction in apoptotic MC3T3-E1 osteoblasts in the p38 MAPK-shRNA transfection group was more pronounced than in the p38MAPK inhibitor group.

Table 3. Percent of apoptosis in MC3T3-E1 cells.

\begin{tabular}{lc}
\hline Group & Apoptosis rate (\%) \\
\hline Normal control & $2.16 \pm 0.40$ \\
High-glucose & $22.62 \pm 3.98^{\Delta}$ \\
p38MAPK-shRNA transfection & $10.16 \pm 2.47^{\Delta * * *}$ \\
p38MAPK inhibitor & $14.21 \pm 2.82^{\Delta *}$ \\
Negative shRNA transfection & $19.64 \pm 3.21^{\Delta}$ \\
\hline Data are reported as means $+\mathrm{SD}$ for $\mathrm{N}=3{ }^{\Delta}{ }^{\Delta} \mathrm{P}<0.01$ vs normal control $* \mathrm{P}<0.05$ vs high-glucose* $* * \mathrm{P}<0.01$ vs
\end{tabular}

Data are reported as means $\pm \mathrm{SD}$ for $\mathrm{N}=3 .{ }^{\Delta} \mathrm{P}<0.01$ vs normal control, $* \mathrm{P}<0.05$ vs high-glucose; $* * \mathrm{P}<0.01 v s$ high-glucose.

\section{Effect of p38MAPK inhibition on p38MAPK protein expression in MC3T3-E1 osteoblasts}

Western blot analysis indicated that p38MAPK expression in MC3T3-E1 osteoblasts in the high-glucose group and in the unrelated shRNA transfection group was clearly increased compared with the normal control group (Figure 3; P $<0.01$ ). Expression of p38MAPK in the p38MAPKshRNA transfection group and in the p38MAPK inhibitor group was significantly decreased compared with that in the high-glucose group $(\mathrm{P}<0.01$ and $\mathrm{P}<0.05$, respectively; Figure 3 ).
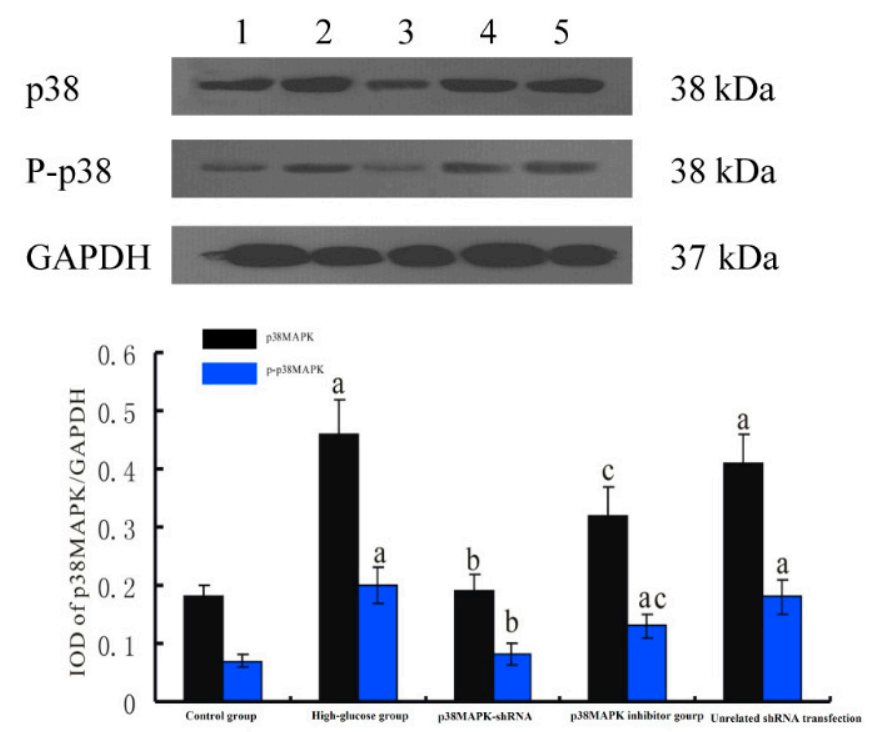

Figure 3. Effect of p38MAPK-shRNA on p38MAPK protein expression in MC3T3-E1 osteoblasts. Lane $1=$ control; lane 2 = high-glucose; lane $3=$ p38MAPK-shRNA transfection; lane $4=$ p38MAPK inhibitor; lane $5=$ unrelated p38MAPK-shRNA transfection. ${ }^{\mathrm{a}} \mathrm{P}<0.01 v s$ control; ${ }^{\mathrm{b}} \mathrm{P}<0.01 v s$ high-glucose; ${ }^{\mathrm{c}} \mathrm{P}<0.05 v s$ high-glucose. 


\section{Effect of p38MAPK inhibition on the binding activities of AP-1 to MC3T3-E1 osteoblasts}

Nucleoprotein extraction showed a clear DNA binding band adding labeled AP-1, while the unlabeled probe competitively inhibited the binding activities of the labeled AP-1 probe, indicating that the EMSA measurement was specific (Figure 4).
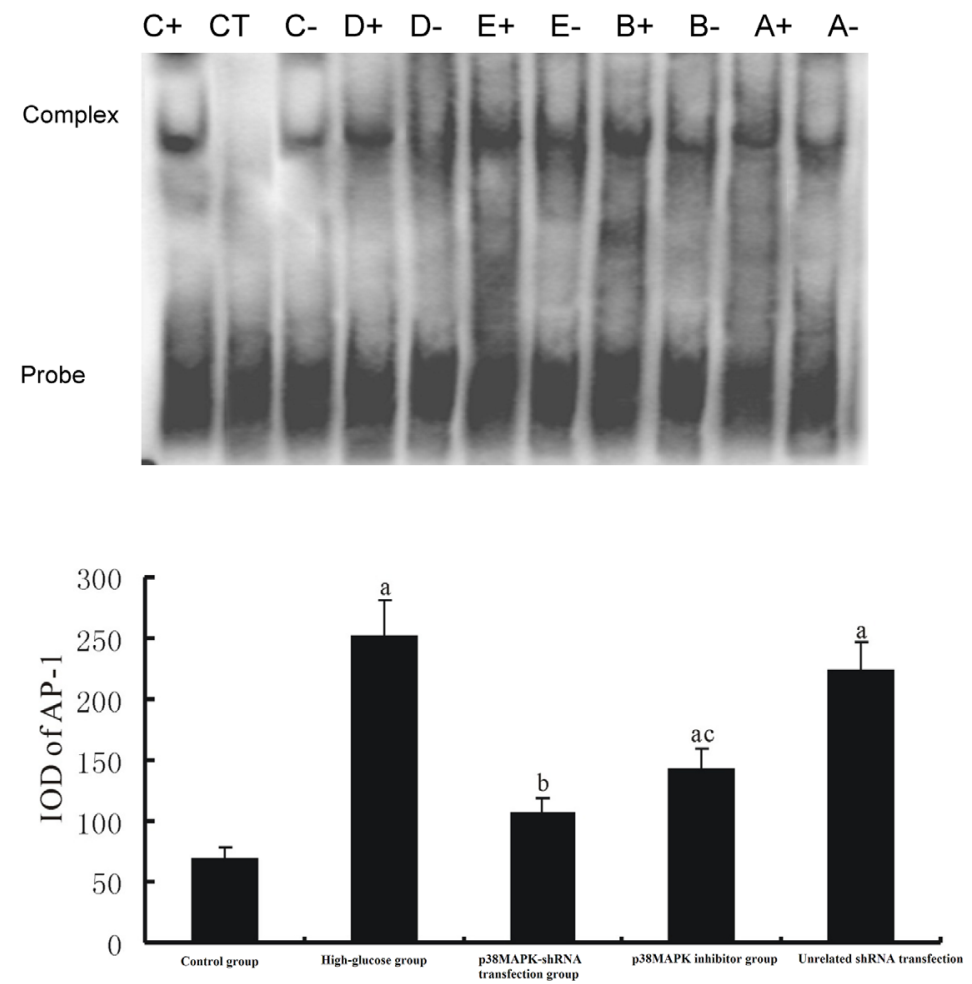

Figure 4. Effect of p38MAPK-shRNA and p38MAPK inhibitor on AP-1 expression in MC3T3-E1 osteoblasts. Upper: $\mathrm{A}=$ control; $\mathrm{B}=$ high-glucose; $\mathrm{C}=\mathrm{p} 38 \mathrm{MAPK}$-shRNA transfection; $\mathrm{D}=\mathrm{p} 38 \mathrm{MAPK}$ inhibitor; $\mathrm{E}=$ unrelated p38MAPK-shRNA transfection. Lower: Normalized data. ${ }^{a} \mathrm{P}<0.01 v s$ control; ${ }^{\mathrm{b}} \mathrm{P}<0.01 v s$ high-glucose; ${ }^{\mathrm{c}} \mathrm{P}<0.05$ $v s$ high-glucose.

The results indicated that AP-1 activity in the high-glucose group and in the unrelated shRNA transfection group was significantly increased $(\mathrm{P}<0.01)$; p38MAPK-shRNA transfection and the p38MAPK inhibitor SB203580 significantly inhibited AP-1 activity with reductions of 57.9 and $45.6 \%$, respectively, compared with the high-glucose group $(\mathrm{P}<0.01$ and $\mathrm{P}$ $<0.05$, respectively; Figure 4).

\section{DISCUSSION}

The formation of bone tissue involves the continuous absorption of old bone tissue by osteoclasts and the reconstruction of new bone tissues by osteoblasts (Mulari et al., 2004; 
Boyce et al., 2012). During bone formation, osteoblasts undergo proliferation, bone matrix maturation, matrix mineralization, and apoptosis, which is regulated by multiple factors (Xing and Boyce, 2005). The apoptosis of osteoblasts is associated with type-I diabetes-induced osteoporosis (Motyl et al., 2012). The basic characteristic of diabetic osteopathia is defective bone formation, as demonstrated by a reduced number of osteoblasts, deficient osteoid formation, slow bone mineralization, and larger bone absorption than bone formation. Hyperglycemia is characteristic of diabetes and is a major cause of diabetic osteopathia; therefore, it is important to understand the pathogenic mechanism of diabetic osteopathia by observing the effect of high glucose on osteoblast apoptosis in vitro. In the present study, we used physiological and high concentration of glucose (5.5 and $22.2 \mathrm{mM}$, respectively) to stimulate the mouse osteoblast strain MC3T3-E1, which retains the morphological and biological characteristics of osteoblasts. The results indicated that high glucose induced apoptosis of the MC3T3-E1 osteoblast cell line, suggesting that hyperglycemia may be one of the mechanisms of diabetic osteopathia. A study of DM and non-DM mice by Lu et al. (2003) indicated that high glucose decreased the expression of osteocalcin, collagen-I, transcription factor Dlx5C-bfal/Runx-2, and the functions of osteoblast to differentiate, proliferate, and secrete osteocalcin and alkaline phosphatase, as demonstrated by a reduction in bone formation. In addition, high glucose can induce apoptosis of endothelia, pancreatic B-cells, epithelia through oxidative stress, AGEs, and phosphokinase $\mathrm{C}$ activation (Jung et al., 2008), suggesting a complicated mechanism for diabetic osteoblastic apoptosis.

As one serine/threonine protein kinase present in multiple types of cells, p38MAPK is an important signaling pathway for nuclear reactions induced by extracellular stimulation and can be activated by multiple stimulators. This pathway regulates cellular biological behaviors by influencing gene transcription and modulation such as cellular inflammatory reactions, proliferation, differentiation, and apoptosis (Wang et al., 2007; Jameel et al., 2009). Recent studies have indicated that the p38MAPK signaling pathway is involved in osteoblast differentiation induced by mechanical stretch (Wang et al., 2012), whereas high glucose can activate the p38MAPK signaling pathway to induce osteoblast apoptosis (Jung et al., 2007). The findings of these studies are consistent with those of the present study indicating that osteoblast apoptosis induced by high glucose is accompanied by activation of p38MAPK, whereas p38MAPK inhibitors attenuate osteoblast apoptosis. This suggests a close correlation between p38MAPK activation and osteoblast apoptosis under high-glucose conditions. However, the mechanism and pathway of apoptosis modulation by p38MAPK is not clear, although some molecules have been implicated in the pathway.

$\mathrm{AP}-1$ is a nuclear transcription factor present as a homogeneous or heterogeneous dimer composed of c-jun and c-fos that binds to DNA through leucine (St-Arnaud and Quelo, 1998). AP-1 binds to a related sequence of target genes, activates the transcriptional activity of target genes, switches the extracellular stimulation signals to nuclear signals, and modulates related gene expression; this modulates cellular proliferation, differentiation, and apoptosis (Liu et al., 1998). The role of AP-1 in osteoporosis is receiving increasing attention (St-Arnaud and Quelo, 1998; Cook et al., 1999; Zhang et al., 2006). The MAPK signaling pathway can influence the activity of AP-1 by increasing AP-1 synthesis or directly inducing AP-1 activity; c-jun can be activated by JNK protein kinase, whereas c-fos can be activated by both JNK and p38MAPK. Lin et al. (2003) found that osteoblast c-fos and c-jun expression is increased in osteoporosis and is related to osteoblast apoptosis, suggesting that AP-1 activation can induce osteoblast apoptosis. To further investigate the roles of p38MAPK and AP-1 in osteoblast 
apoptosis induced by high glucose, we measured the activity of AP-1 in osteoblast MC3T3-E1 cells. The results indicated that high glucose significantly increased AP-1 activities and osteoblast apoptosis, while p38MAPK inhibition attenuated the effect of high glucose. These results suggest that high glucose activates the p38MAPK pathway, which modulates AP-1 activities of MC3T3-E1 and induces apoptosis.

In summary, high glucose induces activation of the p38MAPK pathway, which increases AP-1 activities, and that the signaling pathway is related to apoptosis in MC3T3-E1 osteoblasts. The results obtained provide a theoretical basis for preventing diabetic osteoporosis.

\section{REFERENCES}

Alikhani M, Alikhani Z, Boyd C, MacLellan CM, et al. (2007). Advanced glycation end products stimulate osteoblast apoptosis via the MAP kinase and cytosolic apoptotic pathways. Bone 40: 345-353.

Blakytny R, Spraul M and Jude EB (2011). Review: The diabetic bone: a cellular and molecular perspective. Int. J. Low Extrem. Wounds 10: 16-32.

Boyce BF, Rosenberg E, de Papp AE and Duong le T (2012). The osteoclast, bone remodelling and treatment of metabolic bone disease. Eur. J. Clin. Invest. 42: 1332-1341.

Cook SJ, Aziz N and McMahon M (1999). The repertoire of fos and jun proteins expressed during the G1 phase of the cell cycle is determined by the duration of mitogen-activated protein kinase activation. Mol. Cell Biol. 19: 330-341.

Feng Z, Deng H, Du J, Chen D, et al. (2011). Lentiviral-mediated RNAi targeting p38MAPK ameliorates high glucoseinduced apoptosis in osteoblast MC3T3-E1 cell line. Indian J. Exp. Biol. 49: 94-104.

Inzerillo AM and Epstein S (2004). Osteoporosis and diabetes mellitus. Rev. Endocr. Metab. Disord. 5: 261-268.

Jameel NM, Thirunavukkarasu C, Wu T, Watkins SC, et al. (2009). p38-MAPK- and caspase-3-mediated superoxideinduced apoptosis of rat hepatic stellate cells: reversal by retinoic acid. J. Cell Physiol. 218: 157-166.

Jung DS, Li JJ, Kwak SJ, Lee SH, et al. (2008). FR167653 inhibits fibronectin expression and apoptosis in diabetic glomeruli and in high-glucose-stimulated mesangial cells. Am. J. Physiol. Renal Physiol. 295: F595-F604.

Jung JY, Yoo CI, Kim HT, Kwon CH, et al. (2007). Role of mitogen-activated protein kinase (MAPK) in troglitazoneinduced osteoblastic cell death. Toxicology 234: 73-82.

Lin H, Wei H, Jiang Q and Han Z (2003). Expression of c-fos, c-jun and p53 in bone tissues of primary osteoporosis. Chin. J. Bone Tumor Bone Dis. 2: 368-371.

Liu GY, Frank N, Bartsch H and Lin JK (1998). Induction of apoptosis by thiuramdisulfides, the reactive metabolites of dithiocarbamates, through coordinative modulation of NFkappaB, c-fos/c-jun, and p53 proteins. Mol. Carcinog. 22: 235-246.

Lu H, Kraut D, Gerstenfeld LC and Graves DT (2003). Diabetes interferes with the bone formation by affecting the expression of transcription factors that regulate osteoblast differentiation. Endocrinology 144: 346-352.

Motyl KJ, McCauley LK and McCabe LR (2012). Amelioration of type I diabetes-induced osteoporosis by parathyroid hormone is associated with improved osteoblast survival. J. Cell Physiol. 227: 1326-1334.

Mulari MT, Qu Q, Härkönen PL and Väänänen HK (2004). Osteoblast-like cells complete osteoclastic bone resorption and form new mineralized bone matrix in vitro. Calcif. Tissue Int. 75: 253-261.

Nicodemo AC, Robledo JA, Jasovich A and Neto W (1998). A multicentre, double-blind, randomised study comparing the efficacy and safety of oral levofloxacin versus ciprofloxacin in the treatment of uncomplicated skin and skin structure infections. Int. J. Clin. Pract. 52: 69-74.

Oyama M, Chiba J, Kato Y, Igarashi N, et al. (1998). Distribution and expression of mRNAs for the proto-oncogenes c-fos and c-jun in bone cells in vivo. Histol. Histopathol. 13: 671-678.

Qi JS, Li YN, Zhang BS, Niu T, et al. (2008). Peroxynitrite mediates high glucose-induced osteoblast apoptosis. $J$. Endocrinol. Invest. 31: 314-320.

Shanmugam N, Reddy MA, Guha M and Natarajan R (2003). High glucose-induced expression of proinflammatory cytokine and chemokine genes in monocytic cells. Diabetes 52: 1256-1264.

Smeyne RJ, Vendrell M, Hayward M, Baker SJ, et al. (1993). Continuous c-fos expression precedes programmed cell death in vivo. Nature 363: 166-169.

St-Arnaud R and Quelo I (1998). Transcriptional coactivators potentiating AP-1 function in bone. Front. Biosci. 3: $\mathrm{d} 838-\mathrm{d} 848$.

Tsuchiya T, Tsuno NH, Asakage M, Yamada J, et al. (2008). Apoptosis induction by p38 MAPK inhibitor in human colon cancer cells. Hepatogastroenterology 55: 930-935. 
Wang L, Li JY, Zhang XZ, Liu L, et al. (2012). Involvement of p38MAPK/NF-kappaB signaling pathways in osteoblasts differentiation in response to mechanical stretch. Ann. Biomed. Eng. 40: 1884-1894.

Wang X, Goh CH and Li B (2007). p38 mitogen-activated protein kinase regulates osteoblast differentiation through osterix. Endocrinology 148: 1629-1637.

Xing L and Boyce BF (2005). Regulation of apoptosis in osteoclasts and osteoblastic cells. Biochem. Biophys. Res. Commun. 328: 709-720.

Zhang X, Guo Y, Li R, Zhao Y, et al. (2006). Influence of stretch strain and pressure on expression of osteoblasts' FOS protein. Sheng Wu Yi Xue Gong. Cheng Xue Za Zhi 23: 326-328. 